\title{
Spatial Confinement, Non-Hermitian Hamiltonians and Related Problems
}

\author{
Brian L Burrows ${ }^{1}$, and Maurice Cohen ${ }^{2}$ \\ ${ }^{1}$ Mathematics Section \\ School of Digital Technologies and Arts \\ Staffordshire University, College Road, Stoke-on-Trent,ST4 2DE UK \\ e-mail:brian.burrows2@btopenworld.com \\ ${ }^{2}$ Department of Physical Chemistry \\ The Hebrew University of Jerusalem \\ Jerusalem 91904, Israel \\ e-mail:maurice@fh.huji.ac.il
}

\begin{abstract}
We treat simple examples of systems described by non-relativistic model Hamiltonians which are unconventional. They are not necessarily Hermitian operators. In practice, they often contain applied external fields which are not necessarily small, and they seek to describe the effects of spatial confinement more realistically than most of the classic calculations (some of them by the present authors). A much studied model of a free particle confined by a non-real potential can be accommodated within the same theoretical framework. Numerical treatment of these and similar problems seems well within the capacity of very modest computer systems, as exemplified by a few tabulations.
\end{abstract}

\section{Introduction}

We consider a physical Hamiltonian $\mathcal{H}$, which has real bound states, and the corresponding Schrodinger equation

$$
\mathcal{H} \Psi=E \Psi
$$

together with boundary conditions which ensure that the wavefunction is in the domain of $\mathcal{H}$. These are conditions on the continuity and differentiability and asymptotic conditions so that the wavefunction vanishes at infinite values of the variables. We assume that the domain of the linear operator $\mathcal{H}$ is dense in 
a separable Hilbert space for which an inner product is defined, so that for all $\psi_{1}, \psi_{2}$ in the space the inner product is denoted by $<\psi_{1} \mid \psi_{2}>$. In addition for all $\psi$ in this space

$$
<\psi \mid \psi><\infty
$$

so that $\psi$ can be normalised which implies we are considering bound states and a probability interpretation can be invoked. Given such an inner product we may define an Hermitian conjugate, $\mathcal{H}^{\dagger}$ by means of

$$
<\mathcal{H}^{\dagger} \psi_{1}\left|\psi_{2}>=<\psi_{1}\right| \mathcal{H} \psi_{2}>
$$

Here we consider physical operators $\mathcal{H}$ on the Hilbert space to itself with discrete eigenvalues and corresponding eigenvectors. On any finite subspace of such eigenvectors $\mathcal{H}$ is bounded and consequently the Hermitian conjugate is unique [1].

If $\mathcal{H}^{\dagger}=\mathcal{H}$ then from (1)

$$
E=<\Psi|\mathcal{H} \Psi>=<\mathcal{H} \Psi| \Psi>=<\Psi \mid \mathcal{H} \Psi>^{*}
$$

so that $E$ is real. Physically real energies are necessary for the bound states to be stationary. However although it is sufficient that $\mathcal{H}^{\dagger}=\mathcal{H}$ it is not necessary and real energies may be obtained from non-Hermitian Hamiltonians $[2,3]$. These can arise when the wavefunction is expressed in the modified form $\Psi=\exp (-f) \Phi$, usually to remove a dominant term in the Hamiltonian, and the resulting Schrodinger equation for $\Phi$ involves a non- Hermitian operator in the space. We may also have non-Hermitian operators when $\mathcal{H}$ contains complex terms, which may arise from the interaction of a field ( usually a magnetic field) with an atomic or molecular system.In general the property that $\mathcal{H}$ is Hermitian is independent of the property that the terms in $\mathcal{H}$ are real and non-Hermitian operators exist where all the terms are real.

In this paper we examine techniques for treating such Hamiltonians and illustrate these using model problems. One of these describes the confinement of the hydrogen atom by a harmonic potential, as opposed to an infinite barrier( see [4-12] for some earlier treatments and an extensive review), and we obtain tractable analytic approximations for the wavefunction. All of the necessary integrals are calculated analytically using the properties of the Laguerre polynomials. Another example, where $\mathcal{H}$ contains complex terms, and is a model for the interaction of a particle in a defined state with a magnetic field so that the variable is the spin values of the particle $[13-15,17]$. We also consider a generalisation to multi-states using a well-studied complex valued Hamiltonian $[14,16,17]$, and obtain analytical approximations making use of the properties of the harmonic functions.

Our techniques use an appropriate basis chosen from a complete set in a Hilbert space to form a matrix representation of the operator. The infinite matrix representing the operator in the complete set is considered to be identical to the operator but in some cases a different inner product is used which corresponds to a transformation to an alternative Hilbert space. In the case of confined 
hydrogen we use an alternative inner product in which the operators are Hermitian and we have variational bounds from the matrix analysis. However for the multi-state magnetic problem we use non-Hermitian matrices and we have developed a technique for assessing convergence.

\section{General Theory}

Let $\hat{L}$ denote a linear operator with domain $D_{L}$, which is a subspace of some Hilbert space that can be represented by a matrix operator in a complete set for this subspace. We do not assume that $\hat{L}$ is Hermitian . An important example is where the Hilbert space is the space of square integrable functions $\psi$ so that

$$
\int \psi^{*} \psi d \tau<\infty
$$

In a one-dimensional example in $-\infty<x<\infty$ we have the complete set $\left\{\exp \left(-\alpha x^{2}\right) \omega_{n}(\sqrt{\alpha} x)\right\}$ for any real $\alpha>0$ and where $\omega_{n}(\sqrt{\alpha} x)$ is the nth degree Hermite polynomial.

Another example using a different space, is a model problem where only a finite number of states are considered leading to a finite matrix representation in terms of a set of these functions that form a complete set and hence span the space.

In general, given a specified basis for the domain we may construct a representation matrix A for $\hat{L}$ with elements

$$
A_{i, j}=\mathbf{e}_{i}^{T} A \mathbf{e}_{j}=<\chi_{i} \mid \hat{L} \chi_{j}>
$$

where $\left\{\mathbf{e}_{i}\right\}$ are column vectors with 1 in the ith position and zero elsewhere that are matrix representations of the basis functions $\left\{\chi_{i}\right\}$. The term $<a \mid b>$ denotes the inner product of the Hilbert space and in the special case where $\hat{L}$ is the identity operator, $A$ is the overlap matrix of the basis functions. Assuming that the number of eigenfunctions are in one to one correspondence to the separable basis, which corresponds to the finite matrix not being deficient, we may solve the corresponding matrix eigenvalue equation

$$
A \mathbf{u}_{i}=\lambda_{i} \mathbf{u}_{i} \Rightarrow A U=U \Lambda
$$

where $U$ is a matrix with columns $\mathbf{u}_{i}$ and $\Lambda$ is a diagonal matrix whose diagonal elements are the eigenvalues of $A$ and hence of $\hat{L}$. Thus we can use a similarity transformation to find eigenvalues:

$$
\Lambda=U^{-1} A U
$$

where we assume that if there are degenerate eigenvalues then the columns of $U$ are chosen to be independent vectors. The eigenvalues and eigenvectors may be complex and in general

$$
\mathbf{u}_{i}^{\dagger} \mathbf{u}_{j} \neq 0
$$


so that the eigenvectors are not necessarily orthogonal. But we have

$$
\delta_{i j}=\mathbf{e}_{i}^{\dagger} \mathbf{e}_{j}=\left(U^{-1} \mathbf{u}_{i}\right)^{\dagger} U^{-1} \mathbf{u}_{j}
$$

This shows that the set of vectors $\left\{\mathbf{u}_{i}\right\}$ are orthogonal in a different inner product defined by

$$
\left(\mathbf{u}_{i} \mid \mathbf{u}_{j}\right)=\mathbf{u}_{i}^{\dagger} B \mathbf{u}_{j}=\delta_{i j}, \quad B=\left(U^{-1}\right)^{\dagger} U^{-1}
$$

and that we also have the representation

$$
\left(\mathbf{u}_{i} \mid \hat{L} \mathbf{u}_{j}\right)=\lambda_{i} \delta_{i j}
$$

where the base vectors $\left\{\mathbf{e}_{i}\right\}$ have been replaced by $\left\{\mathbf{u}_{i}\right\}$. Thus $\hat{L}$ is diagonal in this inner product and if the $\lambda_{i}$ are real it is also Hermitian. Any further unitary transformation will preserve the Hermitian property. Thus if the eigenvalues are real then there exists an inner product in which the matrix representation of $\hat{L}$ is Hermitian. If there is only a subset of eigenvalues that are real then there is a subspace for which we can define such an inner product and any subsequent unitary transformation needs to be restricted to this subspace. We note that if $\hat{L}$ is Hermitian in the original matrix inner product then we may choose $U$ to be unitary and then $B$ is the identity and the two inner products are the same.Thus formally $B$ exists but is unknown since it depends on the exact $U$. Examples of this new inner product are given in the following sections including the treatment of a free particle interacting with a given magnetic field. More generally we may define a transformation and the corresponding inner product that simplifies a given non-Hermitian problem. To do this we may use $U_{1}$ whose columns are independent but not eigenvectors of $\mathrm{A}$. We then have

$$
\mathbf{u}_{j}=U_{1}^{-1} \mathbf{e}_{j}
$$

and

$$
\left(\mathbf{u}_{i} \mid \mathbf{u}_{j}\right)=\mathbf{u}_{i}^{\dagger} B_{1} \mathbf{u}_{j}=\delta_{i j}, \quad B_{1}=\left(U_{1}^{-1}\right)^{\dagger} U_{1}^{-1}
$$

but $\hat{L}$ is not diagonal. This is used in a subsequent section to simplify the treatment of confining atomic hydrogen using a harmonic potential by diagonalising a matrix that represents part of $\mathcal{H}$ which contains the kinetic energy terms and consequently we obtain a Hermitian matrix in the new inner product which simplifies the approximations and leads to bounds. In the final section we treat a problem with complex terms in $\mathcal{H}$ which is a model for a particle in the presence of a magnetic field and show that we may choose an inner product that depends on a parameter and that scaling may be carried out to increase the rate of convergence of the eigenvalue problem.

An example which is not a Hermitian representation is the $2 \times 2$ model

$$
A=\left(\begin{array}{cc}
0 & i \omega^{2} \\
-i & 0
\end{array}\right)
$$


where $\omega$ is a real parameter and the eigenvalues are the real quantities $\pm \omega$. This is not unbroken PT symmetric [13] which would be a sufficient condition for real eigenvalues and is considered in the last section. We obtain

$$
U=\frac{1}{\sqrt{\omega^{2}+1}}\left(\begin{array}{cc}
i \omega & -i \omega \\
1 & 1
\end{array}\right)
$$

and we may form the generalised inner product with

$$
B=\frac{\omega^{2}+1}{2 \omega^{2}}\left(\begin{array}{cc}
1 & 0 \\
0 & \omega^{2}
\end{array}\right)
$$

\section{Hydrogen-like atoms in a Harmonic potential}

We consider the radial wavefunction for an hydrogen-like atom confined by a harmonic potential

$$
H=\left(-\frac{1}{2}\left(D^{2}+\frac{2}{r} D\right)-\frac{c}{r}+\frac{b^{2} r^{2}}{2}\right) \psi=E \psi, \quad D=\frac{d}{d r}
$$

The value of the parameter $b$ describes the degree of confinement and differs from more traditional models which usually involve an infinite barrier at some finite value of $r$. The confining potential is therefore a more realistic description of whatever field is used for the confinement and can be regarded as the leading term of a more complicated potential. We require the radial wavefunctions to be continuous and differentiable and that

$$
\int_{0}^{\infty} \psi^{*} \psi r^{2} d r<\infty
$$

so that the wavefunctions belong to the Hilbert space with inner product

$$
\int_{0}^{\infty} \psi_{1}^{*} \psi_{2} r^{2} d r
$$

It is more efficient to use different Hilbert spaces and consequently different sets of basis functions, dependent on the the sizes of $b$ and $c$. To do this we write the wavefunction in terms of an asymptotic factor in the form

$$
\psi=\exp (-f) \phi
$$

where $f=b r^{2} / 2$ or $f=a r$ for large or small $b$ respectively. For large $\mathrm{b}$ the potential is dominated by the harmonic term and this term is always dominant for large $\mathrm{r}$ leading to a discrete spectrum analogous to the harmonic oscillator potential. But for small $b$ the Coulomb potential term dominates for small $r$ so that , particularly for the lower states, the wavefunctions behave analogously to hydrogen-like states and $a$ is related to $c$. These transformations lead to non-Hermitian operators in the inner product in (20) and this corresponds to a similarity transformation of $H$

$$
h=\exp (f) H \exp (-f) \Rightarrow H \exp (-f)=\exp (-f) h
$$


which has the same eigensolution as $H$. The domain of $h$ is the set of functions $\exp (f) \psi$ where $r \psi$ is square integrable. In order to solve the corresponding eigenvalue problem any required boundary conditions on $\phi$ are deduced from those on $\psi$. Thus we may choose the matrix representation

$$
\begin{aligned}
h_{r s}= & <\psi_{r} \mid H \psi_{s}>=\int w \exp (-f) \phi_{r}^{*} H\left(\exp (-f) \phi_{s}\right) d \tau \\
& =<\exp (-f) \phi_{r} \mid H \exp (-f) \phi_{s}>=\left(\phi_{r}, h \phi_{s}\right)
\end{aligned}
$$

where $w>0$ is a chosen weight function and the new inner product to be used is

$$
\left(\phi_{r}, \phi_{s}\right)=\int w \exp (-2 f) \phi_{r}^{*} \phi_{s} d \tau
$$

To use this inner product to calculate estimates to the energies using variational theory we need to choose $f$ so that

$$
\left(\phi_{r} \mid h \phi_{s}\right)=\left(h \phi_{r} \mid \phi_{s}\right)
$$

In general we may write the operator $h$ in the form

$$
h=T+V
$$

where $V$ is a real potential that depends only on the coordinates but $T$ contains derivative terms that arise from the transformation of the kinetic energy terms. Thus a sufficient condition that (25) will hold is that the set of functions $\left\{\phi_{r}\right\}$ are chosen from a complete set so that

$$
\left(\phi_{r} \mid T \phi_{s}\right)=\epsilon_{s} \delta_{r s}
$$

We note that equations (24) and (25) are required for the representation with this new inner product and from section $2, U_{1}$ is the matrix representation of $\exp (f)$. In order to choose $f$ we make use of the results

$$
\begin{gathered}
D \psi=(-\phi D f+D \phi) \exp (-f), \\
D^{2} \psi=\left((D f)^{2} \phi-\left(D^{2} f\right) \phi-2(D f)(D \phi)+D^{2} \phi\right) \exp (-f)
\end{gathered}
$$

In the case where $f=b r^{2} / 2$ the dominant harmonic term cancels and we may write $h=T+V$ where

$$
T=\left(-\frac{1}{2}\left(D^{2}+\frac{2}{r} D\right)+b r D-\frac{3}{2}, \quad V=-\frac{c}{r}\right.
$$

The eigenvalue problem for rT may be written

$$
-2 r T \phi=\left(r D^{2}+2 D-2 b r^{2} D+3 b r\right) \phi=-2 \lambda r \phi
$$

which has the exact solution

$$
\phi_{n}=M\left(-n, \frac{3}{2}, b r^{2}\right)=L\left(n, \frac{1}{2}, b r^{2}\right), \quad \lambda_{n}=2 b n
$$


where $\mathrm{M}$ is the Kummer function and $\mathrm{L}$ the associated Laguerre polynomials which satisfy

$$
\int_{0}^{\infty} L\left(m, \frac{1}{2}, b r^{2}\right) L\left(n, \frac{1}{2}, b r^{2}\right) r^{2} \exp \left(-b r^{2}\right) d r=N_{n} \delta_{m n}
$$

These form a complete set in this Hilbert space ( note $w=r^{2}$ ) and after normalisation to $\phi_{n}$ satisfy the generalised inner product results

$$
\left(\phi_{m} \mid \phi_{n}\right)=\delta m n, \quad\left(\phi_{m} \mid T \phi_{n}\right)=\lambda_{n} \delta m n
$$

so we can choose a basis from this set and we have a diagonal matrix representing T. The remaining matrix has the elements $\left(\phi_{m} \mid V \phi_{n}\right)$ can be calculated analytically summing up the powers of $\mathrm{r}$ and integrating the Gamma functions exactly so as to form Hermitian matrices.For any $b, b \neq 0$ an asymptotic factor is $\exp \left(-b r^{2} / 2\right)$ and all solutions are finite at the origin and satisfy (2) so that they are in the domain of $H$ and in principle this procedure can always be used. However for very small $b$ the asymptotic factor might not be as important so we can consider the alternative method of solution with $f=a r$. In this case we have

$$
T=-\frac{1}{2}\left(D^{2}+\frac{2}{r} D\right)+\frac{a-c}{r}, \quad V=\frac{b^{2} r^{2}}{r}
$$

Analogously the eigenvalue equation for $\mathrm{T}$ can be written

$$
-2 r T \phi=\left(r D^{2}+2 D-2 a r D+a^{2} r+2(c-a)\right) \phi=-2 \lambda r \phi
$$

Choosing $c / a=n+1, n=0,1 \ldots$ then for each separate choice we have the solution

$$
\phi_{n}=M(-n, 2,2 a r)=L(n, 1,2 a r), \quad \lambda_{n}==-\frac{a^{2}}{2}=-\frac{c^{2}}{2(n+1)^{2}}
$$

and $\phi_{n}$ is chosen to be one of the basis functions. For this fixed $a$ the other basis functions are defined as $\phi_{m}=L(m, 1,2 a r)$ so that we use the Hilbert space of these associated Laguerre polynomials which satisfy

$$
\int_{0}^{\infty} L(m, 1,2 a r) L(k, 1,2 a r) \operatorname{rexp}(-a r) d r=N_{m} \delta_{m k}
$$

and the basis can be normalised in this inner product. ( Note $w=r)$. The matrix elements $\left(\phi_{m} \mid V \phi_{k}\right)$ can be calculated exactly using the 3-term recurrence relation for the associated Laguerre polynomials. Thus in all cases we have transformed to a different inner product and hence to different Hilbert spaces but the solutions are in the domain of $H$. When we use $f=a r$, since $a$ depends on a particular $\mathrm{n}$, the chosen basis is suitable for one particular eigenvalue of the original system and only $\phi_{n}$ is a standard solution for a hydrogen-like equation with charge $c$. For $f=b r^{2} / 2$ the same basis can be used to estimate all the states.The difference in these procedures relates to the different dominant terms 
in the potential which approximate a harmonic system or a hydrogen-like system. The convergence and rate of convergence measured by the number of basis functions required supports the different choices of basis functions for large and small $b$.

\subsection{Example Calculations}

In Table1 we present the calculated energies for a range of values of $b^{2}$ for the ground state and first excited state respectively. For the lower values we use the basis $L(n, 1,2 a r)$ and the corresponding inner product whereas for $b^{2}>9$ we use the basis $L\left(n, 1 / 2, b r^{2}\right)$ and the alternative inner product. Two values are given at $b^{2}=9$ so as to compare calculations from the two inner products. In each case we have upper bounds for the energies so that at $b^{2}=9$ we see that the $L(n, 1, a r)$ basis gives slightly better estimates. But for larger $b$ the number of basis elements required is smaller using $L\left(n, 1 / 2, b r^{2}\right)$.

The harmonic potential may be used to model confinement of the hydrogen atom.One advantage is that the potential is effective for all $r$ rather than in the case of an infinite barrier at $r=R$ for some $R$. Previously [11] we calculated the effect of such infinite barriers and for the ground state at $R=1$ we obtained 2.3740 so that this is approximately equivalent to using $b^{2}=9$ in the present calculations but the corresponding potential is not infinite and the wave function is not identically zero near $r=R$. A similar comparison for the first excited state is when $R=1.7$ which approximately corresponds to $b^{2}=3.2$ in the present calculations. 
Table 1: Ground(GS) and First Excited State(ES) Energies

\begin{tabular}{|c|c|c|c|}
$b^{2}(G S)$ & $E_{0}$ & $b^{2}(E S)$ & $E_{1}$ \\
0.0001 & -0.4999 & 0.00001 & -0.1248 \\
0.001 & -0.4985 & 0.001 & -0.1064 \\
0.01 & -0.4857 & 0.0025 & -0.0833 \\
0.1 & -0.3844 & 0.005 & -0.0507 \\
0.35 & -0.1846 & 0.01 & 0.0033 \\
0.5 & -0.0878 & 0.1 & 0.5223 \\
0.75 & 0.0540 & 0.5 & 1.6253 \\
1.0 & 0.1797 & 1.6 & 3.3095 \\
2.0 & 0.5938 & 3.2 & 4.9427 \\
4.0 & 1.2237 & 6.0 & 7.0401 \\
9.0 & 2.3699 & 9.0 & 8.8107 \\
9 & 2.3712 & 9 & 8.8118 \\
16 & 3.5716 & 16 & 12.0596 \\
36 & 6.0676 & 36 & 15.5305 \\
100 & 11.2664 & 100 & 31.9661 \\
2500 & 66.8615 & 2500 & 168.2903
\end{tabular}

\section{Particle in a magnetic field}

Examples of this generalised inner product can be illustrated by considering the problem of a particle interacting with a magnetic field. Initially we consider a particle that in the absence of the field has a particular degenerate energy with two spin states. In the presence of the field the Hamiltonian has the form

$$
\mathcal{H}=E_{0} \mathbf{I}-\mu\left(\sigma_{\mathbf{x}} B_{x}+\sigma_{\mathbf{y}} B_{y}+\sigma_{\mathbf{z}} B_{z}\right)
$$

where $B_{x}, B_{y}, B_{z}$ are the components of the magnetic field, $\mathbf{I}$ is the unit matrix and $\sigma_{\mathbf{x}}, \sigma_{\mathbf{y}}, \sigma_{\mathbf{z}}$ are the Pauli spin matrices ( for spin $\frac{1}{2}$ particles) given by

$$
\sigma_{x}=\left(\begin{array}{ll}
0 & 1 \\
1 & 0
\end{array}\right), \quad \sigma_{y}=\left(\begin{array}{cc}
0 & -i \\
i & 0
\end{array}\right), \quad \sigma_{z}=\left(\begin{array}{cc}
1 & 0 \\
0 & -1
\end{array}\right)
$$

Choosing $E_{0}=r \cos (\theta), B_{z}=\operatorname{irsin}(\theta) \cdot B_{x}=s, B_{y}=0, \mu=-1$ leads to a well known model problem ( [14]-[15] ) given by the $2 \times 2$ matrix representation acting on the vector of spin components

$$
A=\left(\begin{array}{cc}
\operatorname{rexp}(i \theta) & s \\
s & \operatorname{rexp}(-i \theta)
\end{array}\right)
$$

where $r, \theta, s$ are real. This is a non-Hermitian matrix but has PT symmetry. The operator $T$ transforms $i \rightarrow-i$ whereas $P$ is the matrix that interchanges the spin variables given by

$$
P=\left(\begin{array}{ll}
0 & 1 \\
1 & 0
\end{array}\right)
$$


For any vector $\mathbf{x}$ we have

$$
P T A \mathbf{x}=A P T \mathbf{x}
$$

The eigenvalues of $A$ are given by

$$
\lambda=r \cos (\theta) \pm \sqrt{s^{2}-r^{2} \sin (\theta)}
$$

so we obtain a pair of real eigenvalues or a complex conjugate pair depending on the values of the parameters. Application of the PT operators to the eigenvalue equation gives

$$
P T A \mathbf{x}=A P T \mathbf{x}=E^{*} P T \mathbf{x}
$$

so that that $P T \mathrm{x}$ is an eigenvector of $A$ with eigenvalue $E^{*}$. Initially we consider the case of real eigenvalues which is defined by Bender [14] to be unbroken PT symmetry where the action of PT leaves the eigenvalue equation unchanged and a new (positive definite) inner product is constructed to treat this problem where

$$
(u \mid v)=(C P T u)^{\dagger} v=u^{\dagger}\left(T P C^{\dagger} v\right)
$$

and

$$
C=\frac{1}{\cos (\alpha)}\left(\begin{array}{cc}
i \sin (\alpha) & 1 \\
1 & -i \sin (\alpha)
\end{array}\right)
$$

with $\sin (\alpha)=r \sin (\theta) / s$. The matrix of the normalised eigenvectors is

$$
U=\frac{1}{\sqrt{2 \cos (\alpha)}}\left(\begin{array}{cc}
\exp (i \alpha / 2) & \exp (-i \alpha / 2) \\
\exp (-i \alpha / 2) & -\exp (i \alpha / 2)
\end{array}\right)
$$

and consequently

$$
\left(U^{-1}\right)^{\dagger} U^{-1}=\frac{1}{\cos (\alpha)}\left(\begin{array}{cc}
1 & -i \sin (\alpha) \\
i \sin (\alpha) & 1
\end{array}\right)=T P C^{\dagger}
$$

Thus the inner product constructed in [14] for this PT symmetric system is identical with the inner product constructed for a general matrix in the last section. In the case that the eigenvalues are real we have a Hermitian matrix representation. We note that since $U$ is only unique up to normalisation then we may use alternative inner products using different normalisations which will lead to alternative definitions of $C[15]$.

This problem can be generalised to Hamiltonians with multiple energy states in the form

$$
\mathcal{H}=-D^{2} \mathbf{I}-\mu\left(\sigma_{\mathbf{x}} B_{x}+\sigma_{\mathbf{y}} B_{y}+\sigma_{\mathbf{z}} B_{z}\right), \quad D=\frac{d}{d x}
$$

and the components of the field may be chosen as arbitrary functions of $x$. The Hamiltonian is completely defined by taking the domain to be within the space of square integrable functions in $-\infty<x<\infty$ and we require real eigenvalues for bounded stationary states. Here we consider the particular example where $B_{x}=B_{y}=0, B_{z}=i x^{3}, \mu=-1$. This problem is a well-studied problem [14,17] 
and the reality of the eigenvalues has been established ([18],[19]). From (49) we obtain two one-dimensional differential equations:

$$
H \psi=\left(-D^{2}+i x^{3}\right) \psi=E \psi
$$

and

$$
H_{1} \psi_{1}=\left(-D^{2}-i x^{3}\right) \psi_{1}=E \psi_{1}
$$

From the first of these equations (50), writing $\psi=\chi_{1}+i \chi_{2}$ we obtain the two, coupled, real differential equations

$$
\left(-D^{2}-E\right) \chi_{1}-x^{3} \chi_{2}=0, \quad\left(-D^{2}-E\right) \chi_{2}+x^{3} \chi_{1}=0
$$

This is the same pair of coupled equations that need to be solved for (51) if we write $\psi_{1}=\chi_{1}-i \chi_{2}$. Consequently we may obtain the complete solution by solving (50) for bounded wave functions and $E$ real. Thus the domain considered is such that $\psi$ is sufficiently smooth so that it is continuous and differentiable everywhere and is also in the Hilbert space of square integrable functions so that

$$
\psi(x) \rightarrow 0, \quad x \rightarrow \pm \infty
$$

We develop an algorithm for estimating the solutions of (50) and the error in such solutions. We use a matrix calculation with a finite basis of size $N$ chosen from the harmonic functions in the form

$$
\begin{gathered}
\phi_{n}=\exp \left(-\alpha x^{2} / 2\right) \omega_{n}(\sqrt{\alpha} x) N_{n}, n=0,1 . . \\
N_{n}=\frac{1}{2^{2} n !} \sqrt{\frac{\alpha}{\pi}}
\end{gathered}
$$

where $\omega_{n}$ are the nth degree Hermite polynomials and $\alpha>0$ is a chosen nonlinear parameter. For any $\alpha$ this set is a complete orthonormal set for the space. Using the relations

$$
\left.x \phi_{n}=(\sqrt{(} n+1) \phi_{n+1}+\sqrt{n} \phi_{n-1}\right) \frac{1}{\sqrt{2 \alpha}}
$$

and

$$
-\frac{d^{2} \phi_{n}}{d x^{2}}=\left(-\alpha^{2} x^{2}+\alpha(2 n+1)\right) \phi_{n}
$$

we may represent the linear operator $H$ by the matrix $h+i v$ where

$$
h_{m n}=\alpha\left(\frac{1}{2}(2 n+1) \delta_{m, n}-\sqrt{(n+1)(n+2)} \delta_{m, n+2}-\sqrt{n(n-1)} \delta_{m, n-2}\right)
$$

and

$$
\begin{gathered}
v_{m n}=\frac{1}{(2 \alpha)^{3 / 2}}\left\{\sqrt{(n+1)(n+2)(n+3)} \delta_{m, n+3}+\sqrt{(n+1)}(3 n+3) \delta_{m, n+1}+\right. \\
\left.3 n \sqrt{n} \delta_{m, n-1}+\sqrt{n(n-1)(n-2)} \delta_{m, n-3}\right\}
\end{gathered}
$$


We note an important property of these matrices, namely that they are band limited. This will enable an error analysis to be carried out. It is also possible to scale so that $x \rightarrow \gamma x$ so that

$$
\left(h_{N}+i \gamma^{5} v\right) \mathbf{x}=E_{0} \mathbf{x}, \quad E_{0}=E \gamma^{2}
$$

Where $h_{N}$ is the truncated matrix of size $N$ and parameter $\gamma$ can be chosen as small as we like, but for any choice since the matrix is non-Hermitian, we may obtain both real and complex eigenvalues. If we obtain convergence for any real eigenvalue as $N \rightarrow \infty$ then we obtain an eigenvalue of the operator ( or equivalently the infinite matrix in the complete set). It is also possible to vary both $\alpha$ and $\gamma$ as $N$ increases to improve convergence but in the calculations presented here we fix $\alpha=0.5, \gamma=0.5$. The algorithm for estimating the eigenvalues is simply extracting the real eigenvalues from the eigenvalues of (60). We have used Maple to obtain the eigenvalues and the calculation can be done easily and quickly for large N. In the Table 2 we list the estimated eigenvalues where we have increased $N$ until we have convergence to 8 figures. These values agree with those given in [14] to 7 or 8 figures but lower states can be obtained with smaller values of $N$.

Table 2: Calculated Eigenvalues

\begin{tabular}{|c|c|c|}
$\mathrm{n}$ & Energy & $\Delta$ \\
0 & 1.15626707 & $1.0 \times 10^{-21}$ \\
2 & 4.1092288 & $9.7 \times 10^{-21}$ \\
3 & 7.5622739 & $1.9 \times 10^{-19}$ \\
4 & 11.314422 & $8.3 \times 10^{-19}$ \\
5 & 15.291554 & $3.6 \times 10^{-18}$ \\
6 & 19.451529 & $3.4 \times 10^{-17}$ \\
7 & 23.776740 & $5.8 \times 10^{-17}$ \\
8 & 28.217525 & $6.1 \times 10^{-16}$ \\
9 & 32.789083 & $1.2 \times 10^{-15}$ \\
10 & 37.469825 & $7.4 \times 10^{-15}$
\end{tabular}

In order to estimate the error we expand $\psi$ so that

$$
\psi=\sum_{n=1}^{\infty} c_{n} \phi_{n}
$$

and then by truncating $\psi$ we have

$$
H \sum_{n=1}^{N} a_{n} \phi_{n}=\sum_{n=1}^{N} \sum_{m=1}^{N} H_{m n} a_{n} \phi_{m}+\sum_{n=1}^{N} \sum_{m=N+1}^{M+N} H_{m n} a_{n} \phi_{m}
$$

We note that the two terms on the right hand side are orthogonal and the second term, which we denote by $\hat{\chi}$ is orthogonal to the first term. Thus for the exact 
energy $E$ we have

$$
<\hat{\psi}+\hat{\chi}|(H-E) \hat{\psi}>=<\hat{\psi}|(H-E) \hat{\psi}>+<\hat{\chi} \mid \hat{\chi}>
$$

If $\hat{\chi}=0$ we have that the result is exact and we have a matrix operator rather then an operator with an infinite matrix representation. We now choose $\hat{\psi}$ to be a normalised eigenfunction of the matrix $h$ with eigenvalue $E_{0}$ where the elements of $h$ are given by

$$
h_{m n}=H_{m n}, \quad 1 \leq m, n \leq N
$$

then

$$
<\hat{\psi}+\hat{\chi}\left|(H-E) \hat{\psi}>=\left(E_{0}-E\right)+<\hat{\chi}\right| \hat{\chi}>=\left(E_{0}-E\right)+\Delta
$$

and the last term, $\Delta$, is a measure of the truncation effects of representing the operator by a finite matrix. Explicitly we use

$$
\hat{\psi}=\sum_{n=1}^{N} a_{n} \phi_{n}, \quad \chi=\sum_{n=1}^{N} \sum_{m=N+1}^{M+N} H_{m n} a_{n} \phi_{m}
$$

We are not assuming that $H$ is Hermitian so $E_{0}$ may be complex. We may make the following observations from examining $\Delta$

1. If $\Delta$ increases for an identifiable state then this matrix eigenvector is not an approximation to a state of the operator. ( It may vary with $\mathrm{N}$ so much that it is impossible to identify a state with truncation $\mathrm{N}$ with one for truncation $N+1$ but for large $\Delta$ we cannot make any identification with the eigenstate of $\mathrm{h}$ and the eigenstate of $\mathrm{H}$ ).

2. If $\Delta$ decreases as $E_{0}$ converges then we can identify $E_{0}$ as an approximation to some $E$.

3. Since $\Delta$ depends on the eigenvector, then any parameters in the choice of complete set ( such as the exponent in the harmonic basis ) can be chosen to minimise $\Delta$.In the space defined ,(62) is of the required form since the operation on $\psi$ is closed in the space and it is band limited. This property is not an unusual one. One of the simplest forms of a band limited example is a tri-diagonal but in the example considered $M=3$.

The values of $\Delta$, that are also given in Table 2 , are obtained with a maximum size $N=90$ but the numerical process is very quick and the size of the matrix is not a significant problem. However the smaller values in the table can be obtained with smaller eigenvalue calculations. For example for $N=12$ we obtain the lowest state $E_{0}=1.15598023, \Delta=0.00118631$ and for $N=20$ the calculated values are $E_{0}=1.15626805, \Delta=0.0001527$. If however we use $N=50$ then $E_{0}=1.5626707, \Delta=2.29 \times 10^{-13}$. Also in this case the largest absolute value of the eigenvalues is obtained from a complex pair one of which is $E_{0}=11.0605965+i 276.421748, \Delta=142.659$. So we have confidence in the estimate for the ground state but there are complex eigenvalues of the matrix which are not valid estimates of the physical problem. 


\section{Summary}

We have found approximate solutions to model problems for systems which include a confining field. These may be considered as generalising the traditional confinement and do not involve an abruptly rising infinite barrier at a specified point, but rather a more realistic potential which is large in the asymptotic region. One example treats the confinement of hydrogen by a harmonic potential and for the problem of a free particle the imaginary potential confines the particle to a bound state problem. We represent the operators by matrices to obtain accurate estimates of the energies.In all cases the essential property is that we have real bound states and we have illustrated that these can be treated when the given operators are non-Hermitian.

\section{References}

1. J.D.Pryce, Basic Methods of Linear Functional Analysis, ( Hutchinson 1973)

2. A .Mostafazadeh, J. Math. Phys. 43,2814 (2002)

3. A. Mostafazadeh, Pramana J.Phys. 73 , 269 (2009)

4. A Michels ,J. de Boer, A. Bijl, Physica 4, 981 (1937)

5. E. Ley-Koo, S . Rubinstein , J. Chem. Phys. 71,351 (1979)

6 P. W. Fowler ,Mol Phys 53, 865 (1984)

7. C. Laughin, B L Burrows, M Cohen, J.Phys. B 35, 701 (2002)

8 C Laughlin J. Phys .B 37, 4085 (2004)

9. B. L . Burrows, M Cohen, Phys. Rev. A 72, 032508-1 (2005)

10. N .Aquino, G.Campoy, H. E. Montgomery Jr., Int. J. Quant. Chem. 107, 1548 (2007)

11.. B. L .Burrows, M. Cohen, Int.J .Quant. Chem. 106 , 478 (2006)

12. J. Sabin, E.Brandas, S. A .Cruz (Editors), Advances in Quantum Chemistry vol 57, (Elsevier 2009)

13. R. Feynman ,The Feynman Lectures on Physics, Vol 3 , 11-1(Addison Wesley1965)

14. C. Bender ,Rep. Prog. Phys 70947 (2007)

15. F. Kleefeld, arXiv:0906.1011 [hep-th] (2009)

16. C. Bender, S. Boettcher, Phys Rev Lett 80, 5243 (1998)

17. C. Bender, D. C. Brody, H. Jones, Phys Rev Lett 89 , 270401-1 (2002)

18. P. Dorey, C .Dunning, R .Tateo, J.Phys. A 34, L391 (2001)

19. P. Dorey, C .Dunning, R. Tateo, J.Phys A 34, 5679 (2001) 


\section{Acknowledgements}

We wish to place on record our thanks to reviewers, whose helpful comments have led to a greatly improved version of the submitted work. 